\title{
Event-related potentials to overlapping shapes: Effects of saliency and interference
}

\author{
Mathieu B. BRodeur \\ McGill University, Montreal, Quebec, Canada \\ and McMaster University, Hamilton, Quebec, Canada \\ Franco Lepore \\ University of Montreal, Montreal, Quebec, Canada \\ BENOIT A. BACON \\ University of Montreal, Montreal, Quebec, Canada \\ and Bishop's University, Sherbrooke, Quebec, Canada \\ AND \\ Louis Renoult and J. Bruno Debruille \\ McGill University, Montreal, Quebec, Canada
}

\begin{abstract}
Visual perception is often challenged by various difficulties that act concomitantly and whose respective impacts may therefore be hard to distinguish. We used event-related potentials to dissociate the impact of target saliency, generated by occlusion, from that of interference produced by incongruent nontargets. In one block, the target (a square) partially occluded another square tilted by $45^{\circ}$. This nontarget square interfered only to a small extent with target perception. In another block, the target was the occluded stimulus, and interference from the nontarget was substantial. Blocks including two kinds of overlapping shapes (a cross and a square) were added to control for the interference effect. Block comparisons revealed that occlusion modulated an occipital N250 and reaction times. In contrast, interference modulated a parietal N380 but not reaction times.
\end{abstract}

Two of the fundamental, complementary roles of the visual system are to locate specific objects and to evaluate their status and condition. In vision research, these functions are generally tested through the detection of a target presented among distractors and the subsequent discrimination of the specific properties of this target. For the participants to achieve accurate detection and discrimination, the target must be maximally attended, and any nontarget must be ignored. If not adequately ignored, the nontarget can influence the discrimination of the target, but only under the following conditions.

First, the nontarget must resemble the target. For example, a task could require participants to discriminate the direction of a target arrow flanked with nontargets that are also arrows (modified version of the Eriksen \& Eriksen, 1974, task). The correct response will be delivered more slowly if the nontargets are pointing in a direction opposite that of the target (i.e., incongruent condition) than if they are pointing in the same direction (i.e., congruent condition) or in a direction that is not a response option (e.g., up; i.e., neutral condition). Interference causing similar delays of reaction times (RTs) has been found with various stimuli, such as Stroop stimuli (i.e., a color name printed in color; Stroop, 1935), hierarchical stimuli (i.e., a large global letter made up of small local letters; Navon, 1977), and flanker stimuli (i.e., a letter flanked by other letters; Eriksen \& Eriksen, 1974).

The second condition necessary for nontargets to interfere with target processing pertains to the relative visual saliency of these stimuli. The nontargets must be more salient to compensate for the instruction that explicitly invites the participants to ignore them. This compensation is generally mediated by some properties of the nontargets that have visual precedence over the properties of the target. This is well demonstrated with hierarchical stimuli: The global letter has precedence over the local letter (Boer \& Keuss, 1982; Paquet, 1994; Ward, 1983). Interference is indeed greater when the nontarget is global than when this nontarget is local (Navon, 1977). This result suggests that in order to produce interference, nontargets not only need to be incongruous relative to the target, but also need to be more salient than the target.

Interference and saliency's effects are generally measured as a function of the time taken to discriminate the targets, but the brain processes underlying these effects have also been examined using event-related potentials (ERPs).

M. B. Brodeur, mathieu.brodeur@gmail.com 
Conditions of incongruity elicit ERPs with more negative amplitudes somewhere between 200 and $600 \mathrm{msec}$ than congruent conditions (Han \& He, 2003; Han, He, Yund, \& Woods, 2001; Kopp, Rist, \& Mattler, 1996; Liotti, Woldorff, Perez, \& Mayberg, 2000; West \& Alain, 1999). For instance, incongruent hierarchical letters elicit a larger occipitotemporal N2 than do congruent letters (Han, He, et al., 2001), and this effect is known to be larger when the target is local (small letters) than when it is global (large letter; Han \& He, 2003; Han, He, et al., 2001). Despite this body of literature, the way interference interacts with saliency has received little attention and is still unclear. Moreover, ERP studies of interference in which stimuli like the hierarchical and flanker stimuli were used have rarely included a neutral condition. Comparison between congruent and incongruent stimuli might reveal specific effects of interference, but these effects can hardly be dissociated from the facilitation effect induced by congruity.

Our purpose in the present study was to investigate the ERPs to interference and to examine the modulation of these ERPs as a function of the saliency of the nontarget. Interference was induced by an incongruity between the orientations of one square partially occluding another square of the same size but tilted by $45^{\circ}$. The saliency of each shape was determined by whether they were occluded by the other square. When the target was not occludedwhen it was in fact occluding the nontarget - the nontarget was not considered salient, and not much interference was expected. When the target was occluded, the nontarget was salient and should therefore significantly interfere. To isolate the ERPs specific to interference, we added another testing block, in which the target and the nontarget also overlapped, but in which the nontarget was physically different from the target. These stimuli should elicit the ERP effects of occlusion but not those of interference. To better characterize these two ERP signatures, we have also conducted independent-components analyses (ICAs).

\section{METHOD}

\section{Participants}

Twenty right-handed participants (13 female) between 18 and 30 years old took part in the experiment. All of the participants reported, on a written questionnaire, that they had normal or correctedto-normal vision and that neither they nor their first-degree relatives had ever experienced a neurological or psychiatric disorder.

\section{Stimuli}

All of the stimuli are presented in Figure 1. Three stimuli were first created by superimposing a cross over a square, a square over a cross, and a square over a square tilted by $45^{\circ}$. Three additional stimuli were created by tilting each of these stimuli by $45^{\circ}$. The stimuli with interference were the stimuli with two squares. They will be referred to as the same-shape stimuli. The noninterfering stimuli were the stimuli with a cross. They will be designated as the different-shape stimuli. The stimuli were inlaid in a black annulus used to create a condition in Brodeur, Lepore, Bacon, and Debruille (2009). It was preserved here to make the results of the present study comparable to those of the previous one.

\section{Procedure}

The experiment was conducted in a sound-attenuated room. The stimuli were presented in a random order on a computer screen (with a $75-\mathrm{Hz}$ refresh rate) that was placed $60 \mathrm{~cm}$ away from the participants' eyes. All of the stimuli had side lengths covering $12^{\circ}$ of visual angle. Each stimulus appeared for $400 \mathrm{msec}$, with intertrial intervals varying between 1,800 and 2,200 $\mathrm{msec}(M=$ $2,000 \mathrm{msec}$ ). The participants were presented with four blocks of 100 stimuli. The same-shape stimuli were used in two blocks, one with the nonoccluded stimulus as the target (i.e., nonoccluded target) and one with the occluded stimulus as the target (i.e., occluded target). The different-shape stimuli were used in two other blocks. The target was the nonoccluded square in one block and the occluded square in the other block. The instructions for all of the blocks indicated that the participants should discriminate the target by pressing one key of a keyboard when the target was a square and another key when it was a tilted square. The participants were explicitly instructed to perform the task by focusing their attention only on the designated target and to ignore the nontarget. The blocks were administered in a counterbalanced order across participants.

\section{Data Acquisition}

The EEG was captured by 28 tin electrodes placed according to the 10-20 system (American Electrophysiological Society, 1994) on an elastic cap (ElectroCap International). Two additional electrodes were placed on the external canthi and two below each eye to monitor eyeblinks and ocular movements. The impedances in all channels were reduced below $5 \mathrm{k} \Omega$. The signal was referenced to the right earlobe. High- and low-pass filters with half-amplitude cutoffs set at .01 and $100 \mathrm{~Hz}$, respectively, were used in addition to a $60-\mathrm{Hz}$ electronic notch filter. The signals were amplified 20,000 times and digitized at a sampling rate of $1000 \mathrm{~Hz}$.

\section{Data Measures and Analyses}

The signals extracted for analysis were epochs between -200 and $500 \mathrm{msec}$ from stimulus onset. Trials that were contaminated by eye artifacts, excessive myogram, amplifier saturation, or analog-to-digital clipping within the epoch were rejected following an offline automatic rejection procedure. The rejection criteria were analog-to-digital clippings for more than $100 \mathrm{msec}$ and amplitude reaching $\pm 100 \mu \mathrm{V}$. As in Brodeur et al. (2009), there was no significant difference between the ERPs to the square targets and those to the tilted square targets. They were therefore averaged together. This brought the average number of trials per condition to 93 . All of the electrodes were included in the analyses, and they were grouped into
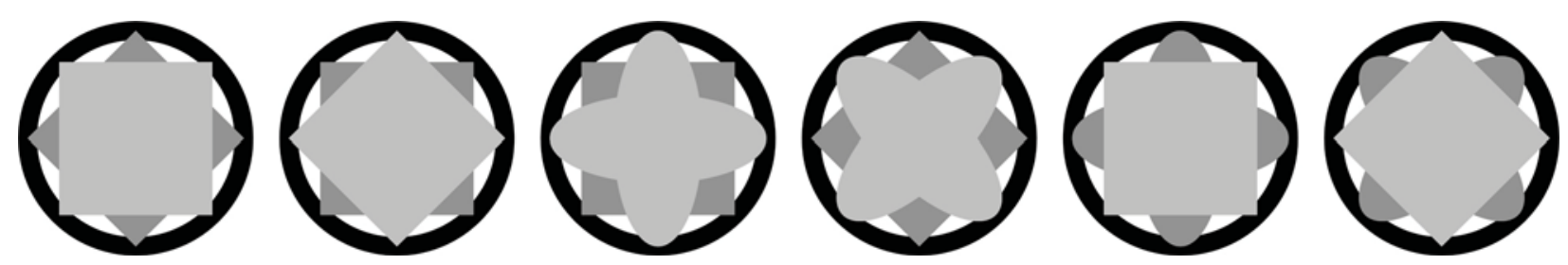

Figure 1. Stimuli of the experiment. The two leftmost are the same-shape stimuli. The four rightmost are the different-shape stimuli. 
seven subsets: occipitotemporal (O1, O2, T5, T6), parietotemporal (T3, T4, Tp7, Tp8), parietal (Pz, P3, P4), centroparietal (Cz, C3, C4, $\mathrm{Cp} 3, \mathrm{Cp} 4)$, frontocentral ( $\mathrm{Fcz}, \mathrm{Fc} 3, \mathrm{Fc} 4)$, frontotemporal (F7, F8, Ft7, Ft8), and frontal (Fz, Fp1, Fp2, F3, F4).

Interference was expected under the conditions that there was an incongruity of orientation between the squares and that the nontarget was more salient than the target. Given that these conditions were both met in the occluded targets of the same-shape stimuli, several comparisons needed to be conducted in order to distinguish their ERP correlates. First, the ERPs to the occluded and nonoccluded targets obtained with the same-shape stimuli were compared with those obtained with the different-shape stimuli. Second, the ERPs to the same-shape and different-shape stimuli were compared for the occluded target and the nonoccluded targets.

Occlusion and shape-similarity differences were analyzed within two successive time windows: that of the N250 and that of the N380, respectively. The latencies of these two time windows were based on the results of our previous study with similar stimuli (Brodeur et al., 2009). In this study, a large negativity to occlusion was found, which encompassed the N250 and the N380 of the present study. It started on average at $212 \mathrm{msec}$ over the occipital electrodes and ended at $466 \mathrm{msec}$ over P3. These two latencies were rounded to 210 and $470 \mathrm{msec}$, respectively. The first latency was used to define the beginning of the time window of the N250, and the second, to end the time window of the N380. We then took into account the fact that the effect modulating the N250 appeared maximal at scalp locations different from those at which the maximum of the effect on the N380 was observed. The first set of locations was occipital, whereas the second was over P3 (see the voltage maps in Figure 3B). We then decided to split the 210- to 470-msec time window into two windows at $325 \mathrm{msec}$, where the N250 stopped decreasing on the subtraction waveforms.

An omnibus repeated measures ANOVA was conducted separately for the two time windows (that of the N250, 210-325 msec; and that of the N380, 325-470 msec) with occlusion (occluded, nonoccluded), similarity (same shape, different shape), and electrode (28 levels) as within-subjects factors. When the interactions were significant, additional analyses were conducted to look for their sources. These analyses were ANOVAs carried out for each electrode subset. The interactions with the electrode factor were computed following an adjustment of the degrees of freedom with the Greenhouse-Geisser (1959) correction. They will be presented with the original degrees of freedom, the epsilon value reflecting the heterogeneity of variance, and the corrected $p$ values.

\section{ICA}

An ICA was used to determine whether the components that made the largest contribution to the ERPs in the time window of the N250 were different from those that contributed most to the N380 time window. The grand average subtraction ERPs (nonoccluded target - occluded target of the same-shape condition) of 29 channels ( 28 electrodes and 1 channel monitoring vertical eye movements) in an epoch of -200 to $700 \mathrm{msec}$ (901 time points) were submitted to ICA. ICA decomposes the ERPs into a sum of sparsely activated independent components (ICs) with fixed scalp maps and maximally independent time courses (Makeig, Jung, Bell, Ghahremani, \& Sejnowski, 1997). The ICA was conducted with EEGLAB Version 6.01b (Delorme \& Makeig, 2004), a freely available open-source toolbox (www.sccn.ucsd.edu/eeglab) running under MATLAB Version 7.7 (The MathWorks, Natick, MA). More specifically, we applied infomax ICA (Bell \& Sejnowski, 1995) with the EEGLAB function runica (Delorme \& Makeig, 2004), producing 29 ICs. We then used the envtopo() function of EEGLAB to identify the ICs that together accounted for at least $80 \%$ of the variance (PVAF) of the ERP effect in the N250 time window (210$325 \mathrm{msec}$ ) and those accounting for the same amount of variance in the N380 time window (325-470 msec; Delorme \& Makeig, 2004; Onton, Westerfield, Townsend, \& Makeig, 2006).

\section{RESULTS}

\section{Behavioral Results}

The rate of errors was $3 \%(S D=3)$ in all conditions except in the nonoccluded target condition with sameshape stimuli, in which it was $2 \%(S D=2)$. No statistical analysis was conducted because of the very low value of these rates and the likeliness of a floor effect. Mean RTs with the different-shape stimuli were $591 \mathrm{msec}(S D=75)$ for the nonoccluded target and $621 \mathrm{msec}(S D=74)$ for the occluded target. With the same-shape stimuli, they were of $587 \mathrm{msec}(S D=70)$ for the nonoccluded target and $617 \mathrm{msec}(S D=72)$ for the occluded target. Significance was achieved only for the occlusion factor, meaning that the discrimination of a target was slowed down when it was occluded by the nontarget $[F(1,19)=13.82, p<$ $.001]$. On the other hand, RTs did not change between the same- and the different-shape stimuli, meaning that similarity had no impact on RTs.

\section{ERPs}

Figures 2A and 2B depict the ERPs to the same-shape (Figure 2A) and different-shape (Figure 2B) stimuli. The ERPs to the occluded target started to be more negative than the ERPs to the nonoccluded target at $210 \mathrm{msec}$. This effect peaked at $250 \mathrm{msec}$ and ended at $325 \mathrm{msec}$ with the different-shape stimuli. It was consequently labeled the N250. The negativity lasted much longer with the sameshape stimuli and ended at $470 \mathrm{msec}$. Figure $3 \mathrm{~A}$ depicts the subtraction data between the occluded and nonoccluded targets of the same-shape stimuli. It suggests that the longer latency to the negativity with the same-shape stimuli was due to another component, which peaked at $380 \mathrm{msec}$. It can be seen that the 210 - to 470 -msec negativity was made up of an N250 peaking at the occipital electrodes and of a second negativity, the N380, peaking around $380 \mathrm{msec}$ at parietal electrodes. The voltage maps of the N250 and N380 in Figure 3B illustrate the respective occipital and parietal distributions of these two components. Note that the P1 difference that can be detected by visual inspection of the ERPs to the same-shape stimuli was tested with a 40msec time window centered on the peak of the difference but was not statistically significant.

The ANOVA conducted on the mean voltage of ERPs in the 210 - to $325-\mathrm{msec}$ time window for the 28 electrodes led to an interaction of the three factors $[F(27,513)=$ $4.97, p<.001, \varepsilon=.203]$ but, most importantly, the occlusion and similarity factors did not interact $[F(1,19)=$ $0.93, p=.764]$. The same ANOVA model was applied to each subset of electrodes. The results indicated that the ERPs to the occluded target were significantly more negative than those to the nonoccluded target at the frontocentral $[F(1,19)=7.84, p=.011]$, centroparietal $[F(1,19)=$ $11.3, p=.003]$, parietal $[F(1,19)=13.5, p=.002]$, parietotemporal $[F(1,19)=7.79, p=.012]$, and occipitotemporal $[F(1,19)=9.30, p=.007]$ subsets. The differences were not significant at the frontal and frontotemporal subsets. There was no significant interaction between occlusion and similarity at any subset. 
A
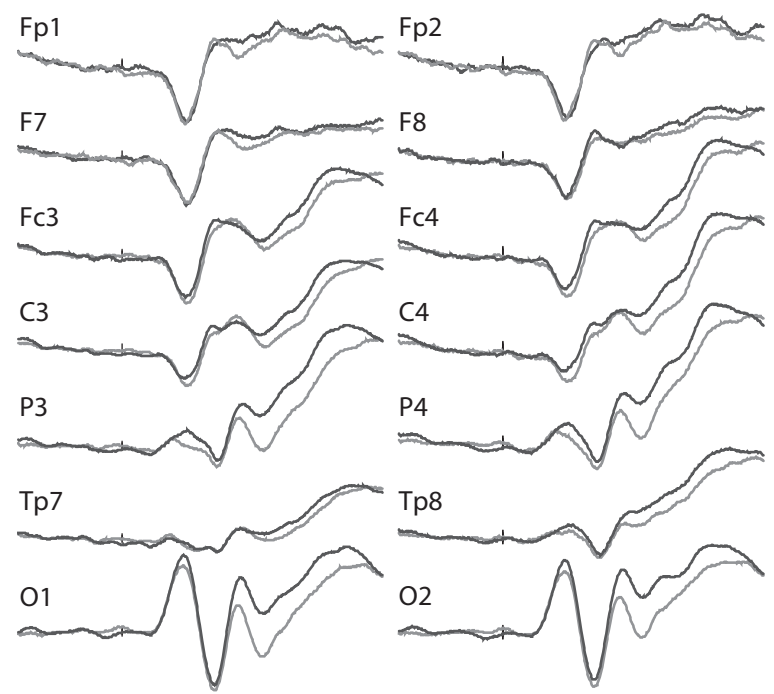

Same-Shape Stimuli
B

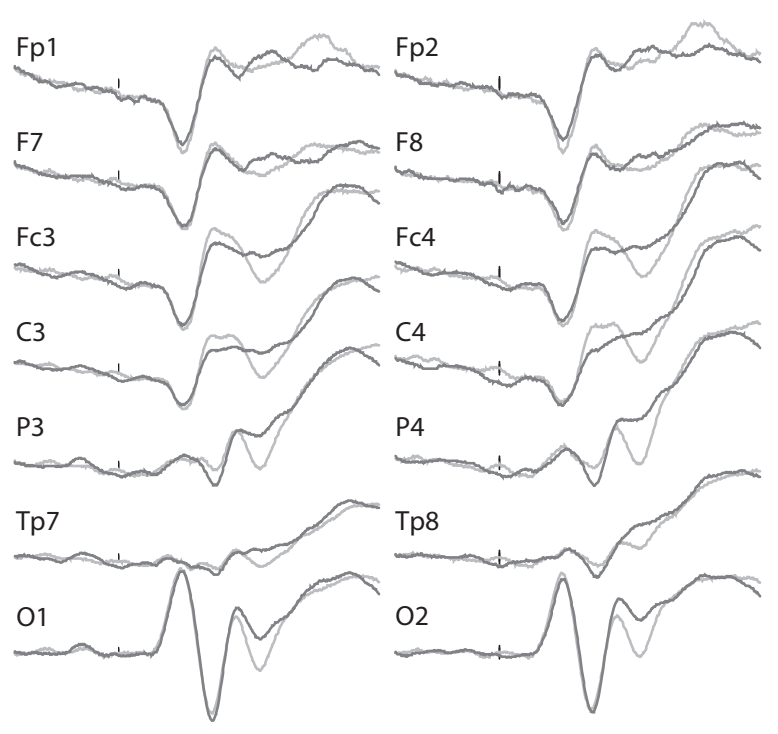

Different-Shape Stimuli

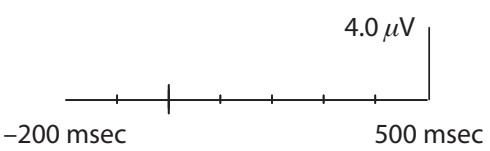

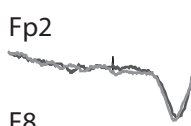

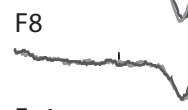

Fc4

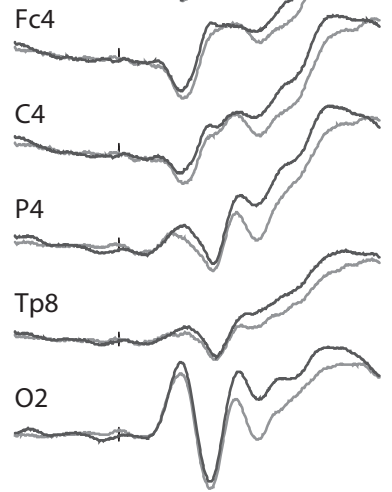
porat
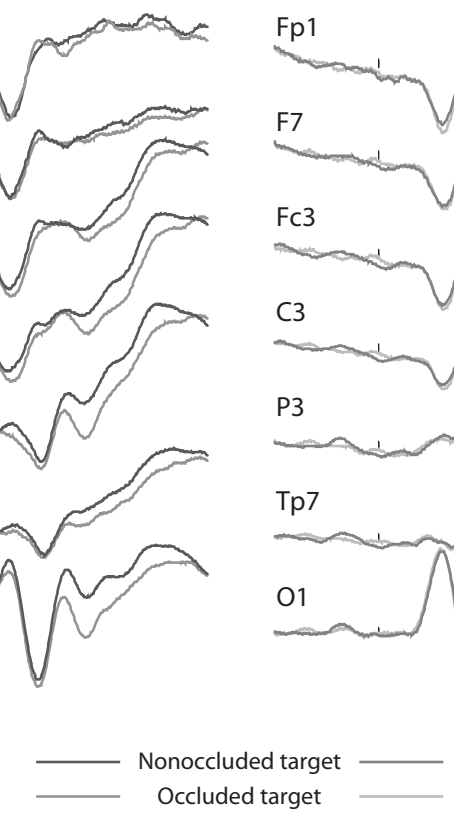

Figure 2. Comparisons of the grand averages of the ERPs to the occluded and nonoccluded conditions with the same-shape (A) and different-shape $(B)$ stimuli $(n=20)$. Each electrode subset is represented by one electrode from each hemiscalp (Fp2/Fp1 for the frontal, F8/F7 for the frontotemporal, Fc4/Fc3 for the frontocentral, C4/C3 for the centroparietal, P4/P3 for the parietal, Tp8/Tp7 for the temporoparietal, and $\mathrm{O2} / \mathrm{O1}$ for the occipitotemporal subset).

In the time window of the N380, the three-way interaction was barely significant $[F(27,513)=2.33, p=.052$, $\varepsilon=.172]$. The mean amplitude was more negative for the occluded target than for the nonoccluded target with the same-shape stimuli $[F(1,19)=7.10, p=.015]$ but not with the different-shape stimuli $[F(1,19)=0.173, p=.682]$. The analyses for each subset showed that there was an occlusion effect but no interaction at the occipitotemporal subset $[F(1,19)=7.93, p=.011]$. More importantly, the occlusion and similarity factors interacted at the parietal $[F(1,19)=4.45, p=.020]$ and centroparietal $[F(1,19)=$ $4.31, p=.050]$ subsets. A three-way interaction was also found at the parietotemporal subset $[F(3,57)=5.26, p=$ $.008, \varepsilon=.705]$. There was no significant occlusion effect or interaction at the frontal, frontocentral, or frontotemporal subset.

The scalp distributions of the N250 and N380 effects were slightly lateralized. To test this asymmetry, we completed additional analyses from which the midline electrodes (Fz, Fcz, Cz, and Pz) were excluded and in which the hemiscalp factor (left, right) was added. The results indicate that for the $\mathrm{N} 250$, there was a hemiscalp $\times$ similarity $\times$ occlusion interaction at the parietotemporal subset $[F(1,19)=5.36, p=.032]$ and a nearly significant fourway interaction at the frontotemporal subset $[F(1,19)=$ $3.46, p=.078]$. Further analyses showed that the hemiscalp factor interacted marginally with the occlusion factor in the different-shape $[F(1,19)=4.51, p=.047]$ and the same-shape $[F(1,19)=3.68, p=.053]$ stimuli. Analyses carried out for the $\mathrm{N} 380$ revealed a hemiscalp $\times$ similarity $\times$ occlusion interaction only at the parietotemporal subset $[F(1,19)=8.42, p=.009]$.

To test the N380 and its relationship to the similarity factor, we conducted two similarity $\times$ electrode ANOVAs: one with the occluded target and one with the nonoccluded target. The interaction was significant with the occluded target $[F(27,513)=4.39, p=.003, \varepsilon=.146]$. The analyses conducted on each subset indicated that the similarity effect was significant at the parietal subset $[F(1,19)=$ $4.81, p=.041]$ and was nearly significant at the central subset $[F(1,19)=3.24, p=.088]$. The N380 was therefore significantly larger for the occluded target of the sameshape stimuli than for the occluded target of the differentshape stimuli over the parietal area. This effect can be seen in Figure 4A. The same analyses were conducted in the time window of the N250 to ascertain that the difference between same- and different-shape stimuli was restricted to the N380. No main effects or interactions reached significance in these analyses. Moreover, there was no significant interaction or main effect of similarity with the nonoccluded target. This is consistent with Figure 4B, which depicts N380s that are mostly identical across conditions.

An ANOVA with time window (N250, N380), electrode (28 levels), and occlusion as within-subjects factors was carried out on the ERPs to the same-shape stimuli. There was a three-way interaction $[F(27,513)=3.47, p=.012$, 


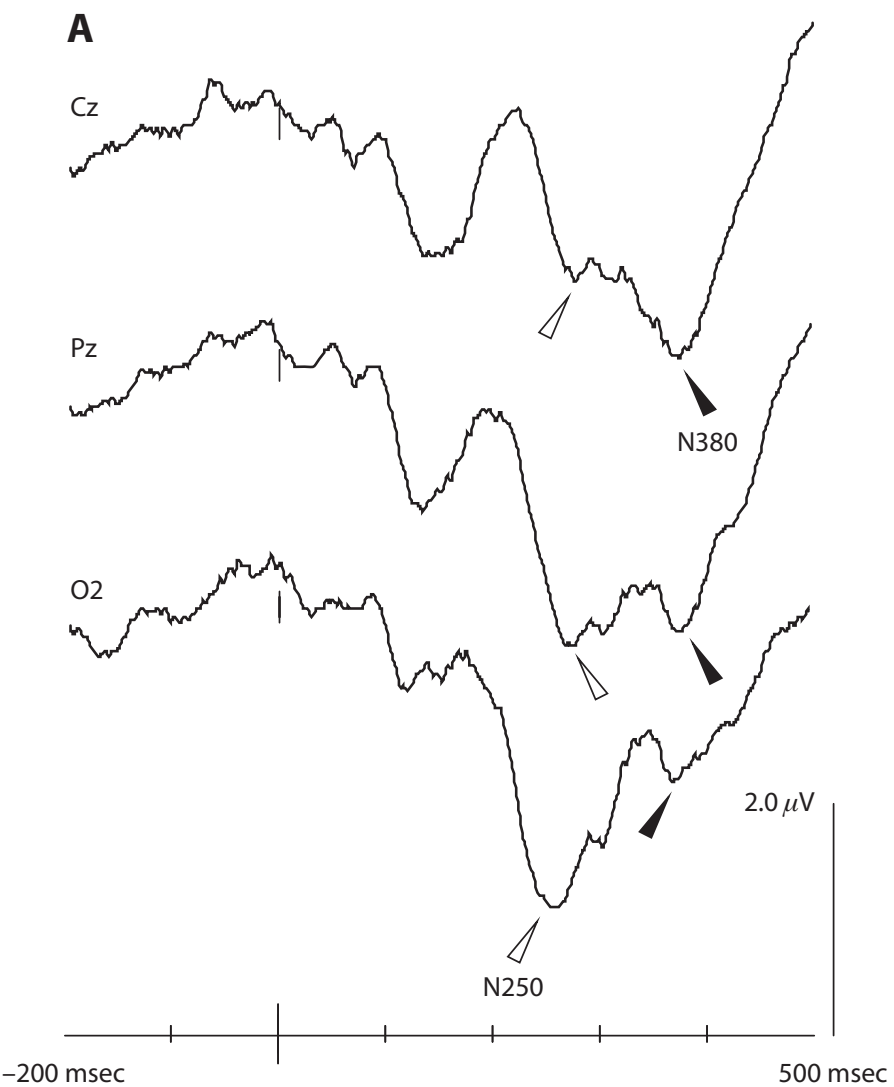

\section{B $\quad \mathrm{N} 250(210-325 \mathrm{msec})$}

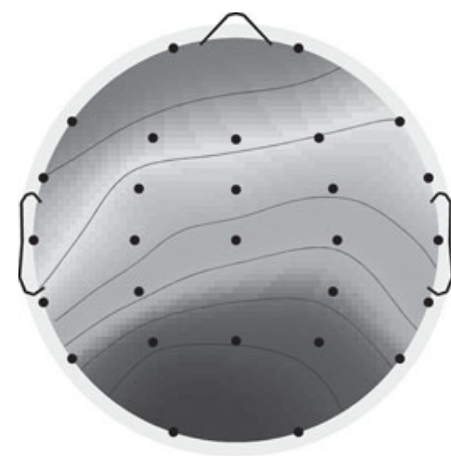

N380 (325-470 msec)

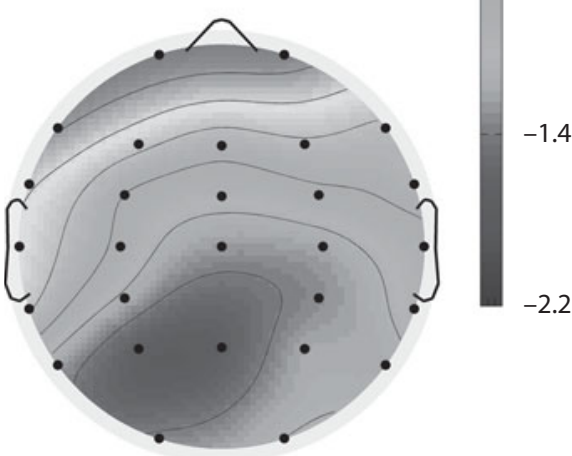

Figure 3. (A) Results of the subtractions of the ERPs to the nonoccluded target from the ERPs to the occluded target with the sameshape stimuli at $\mathrm{O} 2, \mathrm{Pz}$, and $\mathrm{Cz}$. The $\mathrm{N} 250$ and $\mathrm{N380}$ effects are identified with white and black arrowheads, respectively. (B) Voltage maps of these effects.

$\varepsilon=.149]$. The $\mathrm{N} 250$ and the N380 therefore had significantly distinct scalp topographies, as was suggested by the subtraction data presented in Figure 3A. The results from the ICAs, displayed in Figure 5, show that five ICs contributed to $83.4 \%$ of the variance of the $\mathrm{N} 250$ and to $86.0 \%$ of that of the N380. Four out of the five components that contributed the most to each effect were the same: IC1, IC2, IC4, and IC6 accounted for $76 \%$ of the N250 and $80 \%$ of the N380. Note, however, that all of these components did not contribute to the same extent to each effect. Although IC2 and IC6 made comparable contributions to the two effects (IC2, 24\% of the variance of the N250 and $29 \%$ of the variance of the N380; IC6, $21 \%$ of the variance of the $\mathrm{N} 250$ and $22 \%$ of the variance of the N380), IC1 contributed more to the N250 (50\%) than to the N380 (33\%), and IC4 contributed much less to the N250 (6\%) than to the N380 (25\%). Moreover, one of the five most contributing components, IC14, contributed only to the N250 effect ( $10 \%$ of the variance), and another, IC9, contributed only to the N380 effect (17\% of the variance). The leftward shift in the voltage distribution of the ERPs to the same-shape stimuli between the two time windows (see Figure 3B) could be due to the greater contribution of IC4 to the second effect, because this IC had a left temporoparietal scalp distribution.

\section{DISCUSSION}

The present study shows that the processing of the shared property (i.e., the square shape) and the processing of saliency are indexed by two different ERP components. We first showed that ERPs were more negative over posterior scalp sites when nontargets were similar to targets and more salient than them. This effect started around $210 \mathrm{msec}$ and ended around $470 \mathrm{msec}$. These results are globally consistent with modulations that have been reported in other conditions of interference (Han \& He, 2003; Han, He, et al., 2001; Kopp et al., 1996; Liotti et al., 2000; West \& Alain, 1999). More analyses showed that the first part of this long negativity, the N250, was specifically modulated by the occlusion factor that was used to manipulate target-nontarget relative saliency. This effect was larger over the occipital area and, more particularly, over the right hemiscalp. Occlusion also affected RTs, indicated by the longer RTs to the occluded than to the nonoccluded targets. The second part of the ERP effect, the parietal N380, was exclusively modulated by the similarity factor or, more specifically, by the interference generated by nontargets that had the shape of the target but that were oriented differently. This effect was greater over the parietal area and, more specifically, over the left 
A
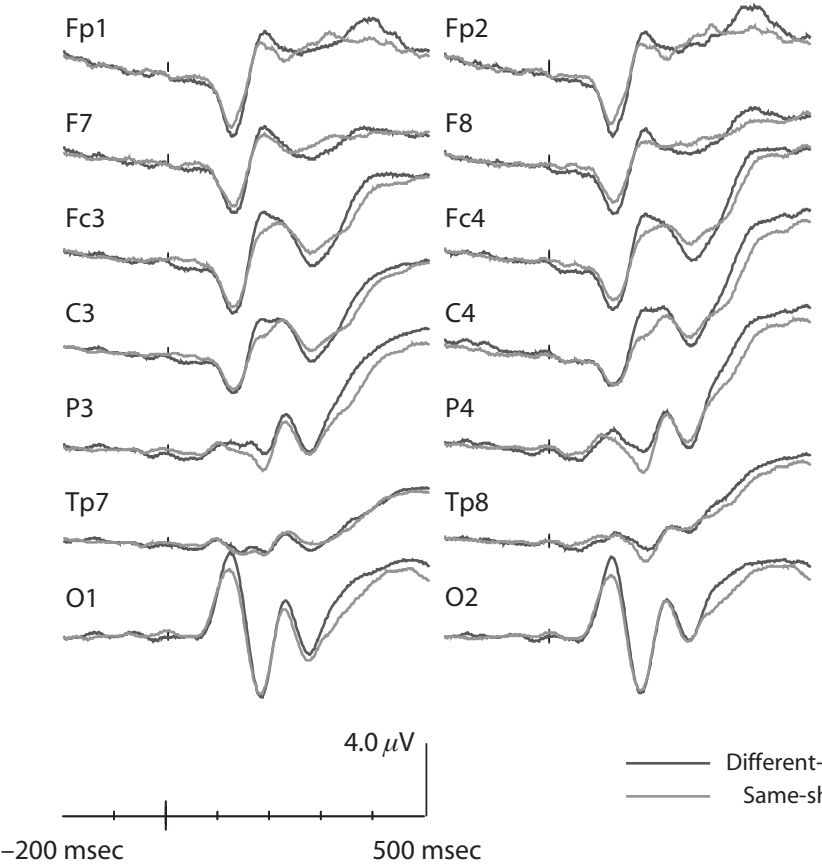

B Nonoccluded Target

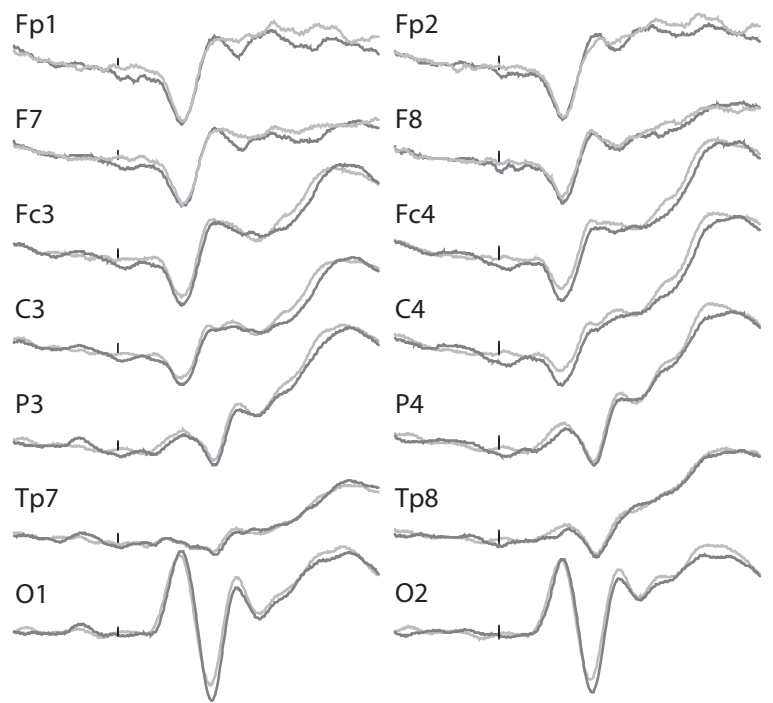

Figure 4. Comparisons of the grand averages of the ERPs to the same-shape and different-shape stimuli in the occluded (A) and nonoccluded $(B)$ conditions $(n=20)$. Each electrode subset is represented by one electrode from each hemiscalp (Fp2/Fp1 for the frontal, F8/F7 for the frontotemporal, Fc4/Fc3 for the frontocentral, C4/C3 for the centroparietal, P4/P3 for the parietal, Tp8/Tp7 for the temporoparietal, and $\mathrm{O} 2 / \mathrm{O} 1$ for the occipitotemporal subset).

hemiscalp. Interestingly, similarity did not increase the RTs needed to discriminate the target.

The N250 and N380 had mostly the same ICs. The results showed that four out of the five components that contributed the most to the effect were the same for these two negativities. The difference between the scalp distribution of the N250 and that of the N380 may be due to the component that was specific to each of these negativities - that is, IC14 for the N250 and IC9 for the N380. A closer look of Figures $3 \mathrm{~B}$ and 5 shows that the voltage map of IC14 best matches the voltage map of the N250. The four other components - that is, those that were common - may have also contributed to this scalp distribution difference, because they contributed to the N250 and N380 to different extents. Three of these four common components had a bilateral posterior scalp distribution, whereas the last one (IC4) had a left centroparietal distribution. One of the posterior components, IC1, contributed significantly more to the N250 than to the N380, whereas the centroparietal component contributed much more to the N380 than to the $\mathrm{N} 250$. The mechanisms activated in response to interference might therefore be generic, but the extent of their solicitation probably depends on the specific conditions causing this interference.

\section{N250 and Occlusion Processing}

Larger N250s reflect greater allocation of brain resources to process a salient nontarget and/or to compensate for weaker perception of the target. Greater alloca- tion of resources to a salient nontarget happens because this nontarget is complete and has more visible parts than the target. However, these resources may also be useful to the perception of the occluded target (Brodeur et al., 2009), which needs to be amodally completed. Amodal completion is the process that brings together the disparate pieces of objects and provides them with the impression of a unitary surface placed underneath an occluder (Wagemans, van Lier, \& Scholl, 2006). This impression, which can only be achieved by processing the relative depth of an occluder and an occluded object (Kellman \& Shipley, 1991), significantly favors the perception of the incomplete object (Brown \& Koch, 1993; Gegenfurtner, Brown, \& Rieger, 1997). From a different point of view, it could also be argued that the occlusion creates interference by deviating attention away from the target. This possibility has already been put forward to account for the earlier part of the N2 effect elicited by the incongruent condition in the flanker task (Gehring, Gratton, Coles, \& Donchin, 1992; Kopp et al., 1996). In any case, amodal completion and interference both imply that the ratio of target to nontarget saliency is diminished, and this, in turn, can easily account for the longer RTs obtained for the occluded than for the nonoccluded stimuli.

Alternatively, one may reason that the N250 is unrelated to the perception of the occluder but, rather, reflects additional resources specifically allocated to compensate for the incompleteness of the target. In support to this argument, Doniger et al. (2000) showed that fragmenting 


\section{N250 (210-325 msec)}

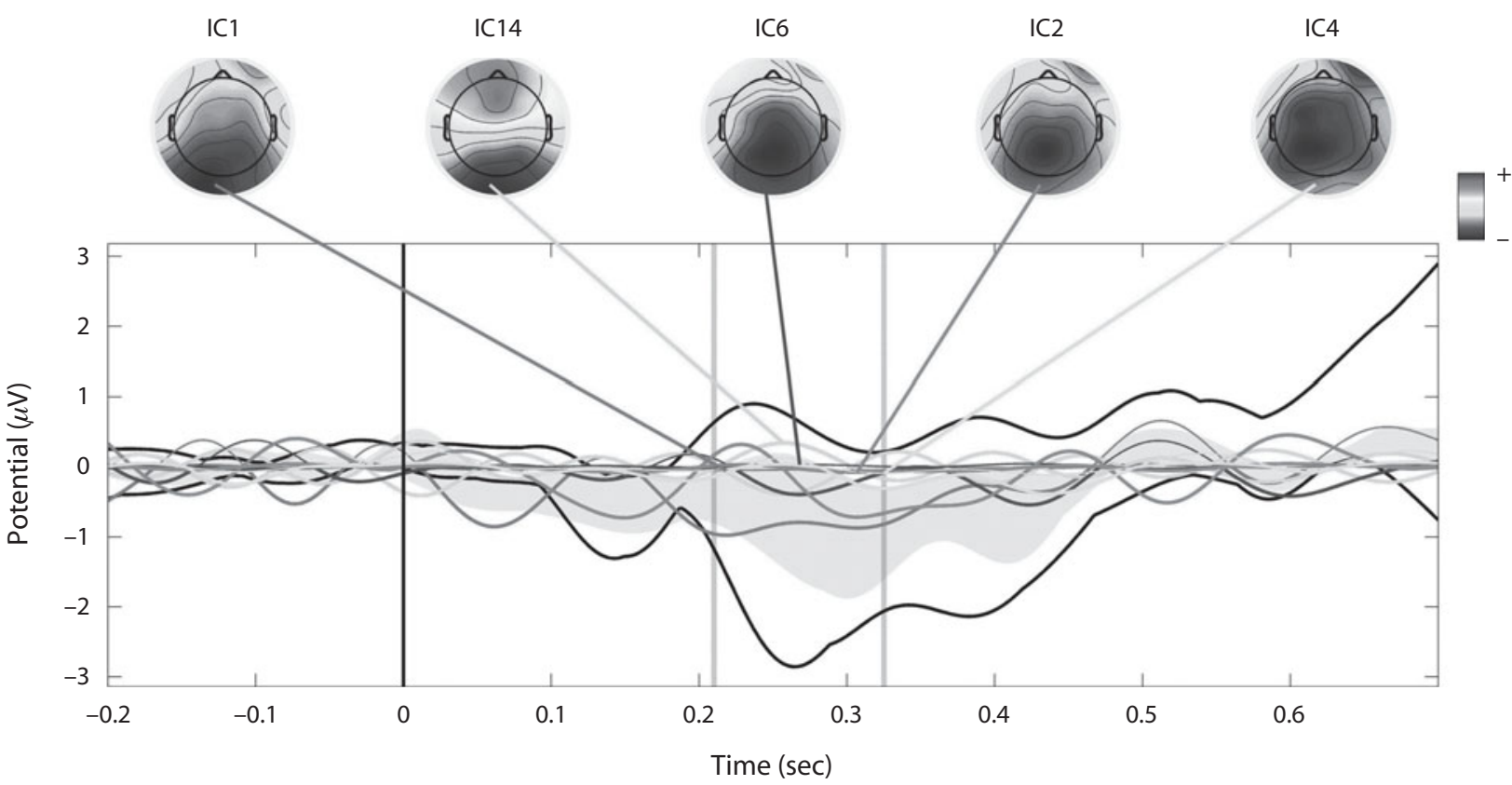

N380 (325-470 msec)

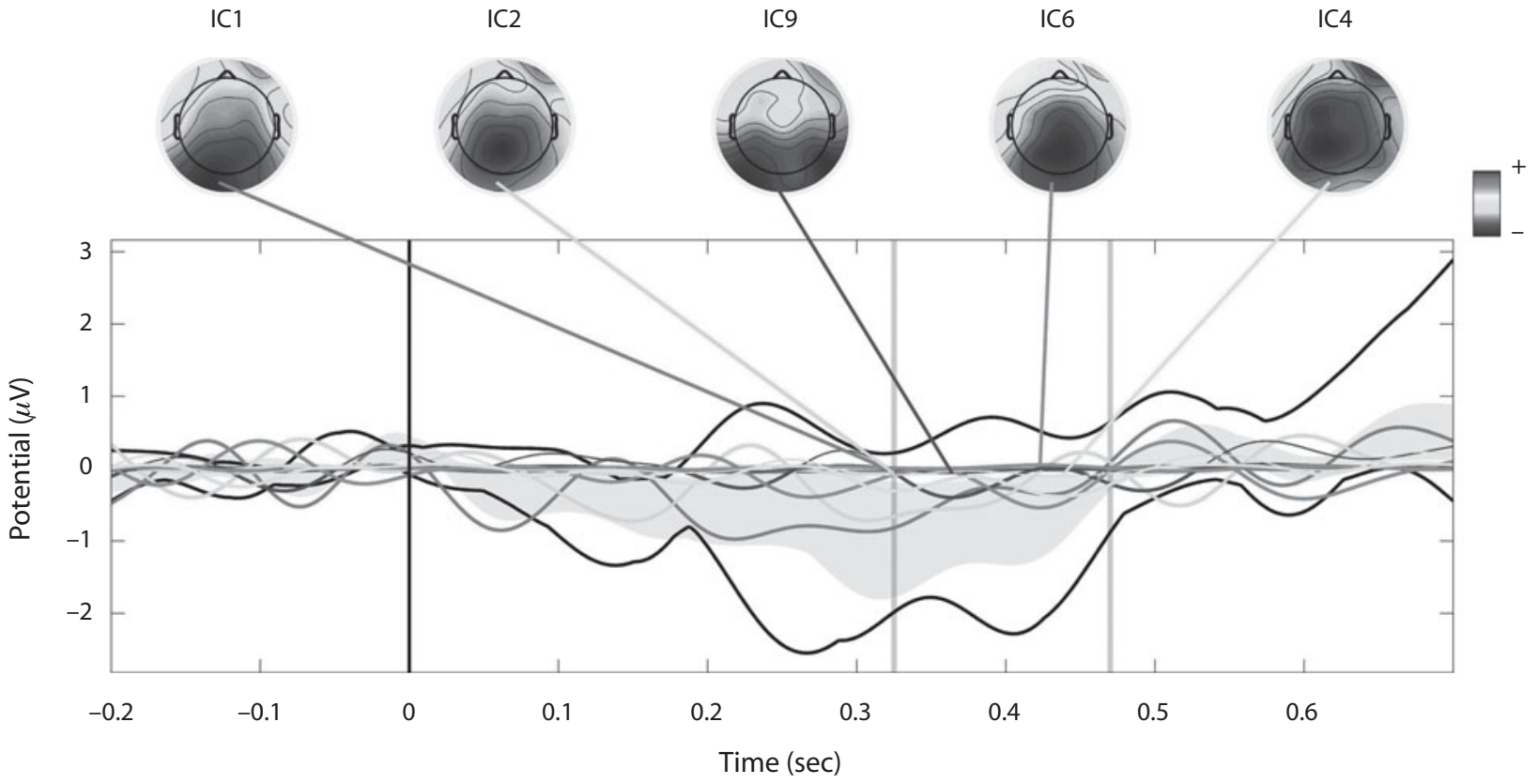

Figure 5. The five independent components (ICs) contributing the most to the $\mathbf{N 2 5 0}$ and $\mathbf{N 3 8 0}$ effects (subtraction data). The vertical black lines at time 0 indicate target onset. The thick black lines show the envelope, which are the most positive and negative values of the ERPs over all channels and at each time point. The head traces show the envelopes of the contribution of the ICs represented. Each IC scalp map is connected to its data envelope by a gray line that points to the moment of that component's peak contribution to the ERP (see Delorme \& Makeig, 2004).

pictures of objects elicits a negativity that is comparable to the N250. This negativity, the Ncl (i.e., negativity to closure) started at $230 \mathrm{msec}$ and peaked at $290 \mathrm{msec}$ bilaterally over the occipitotemporal scalp region. It was associated with closure mechanisms by which missing information was filled in to allow for stimulus identification. Indeed, closure does not need incomplete objects to be partially occluded and, therefore, does not activate amodal completion. The possibility that the N250 reflects closure is nevertheless undermined by the results from our 
previous study (Brodeur et al., 2009). In this study, one of the nonoccluded shapes was a white square overlapping a gray square. Because this square was presented on a white background, it was incomplete and had only the parts overlapping the black annulus and the occluded square as visible contours. Despite its incompleteness, this square created interference over the occluded square, reflected by longer RTs and a negativity starting as soon as $206 \mathrm{msec}$ over $\mathrm{O} 2$ when the target was the occluded square (Brodeur et al., 2009). If incompleteness alone was responsible for the N250, no difference would have been observed between the occluded and nonoccluded conditions, because they were both incomplete. In fact, the evidence indicates that completion of easily identifiable objects, such as this white square, is processed earlier than the N250, over the N1 or even the P1 (Brodeur, Lepore, et al., 2008; Murray, Foxe, Javitt, \& Foxe, 2004; Pegna, Khateb, Murray, Landis, \& Michel, 2002).

\section{N380 and Interference}

The ERP effects related to target-nontarget similarity peaked around $380 \mathrm{msec}$. They reflect the brain response to interference. Of great importance is the fact that the N380 was greater for the same-shape stimuli than for the different-shape stimuli only when the target was occluded by the nontarget (compare Figure 4A with Figure 4B, for which the target was not occluded). This result implies that the similarity of the nontarget to the target is not sufficient to elicit ERP interference effects. The source of the interference also needs to be more salient than the target, which was obtained here by placing the nontarget in front of the target. The modulation of the N380 by saliency was consistent with observations in other studies in which target-nontarget relative saliency was also manipulated. For instance, Han and colleagues (Han \& He, 2003; Han, Song, Ding, Yund, \& Woods, 2001) reported that the temporooccipital N2 to interference was diminished after the local target of a hierarchical stimulus was made more salient by coloring it. Similarly, Kopp et al. (1996) showed that the N2 effect associated with interference was reduced when the nontargets flanking the target were slightly moved away from that target. Coloring a target and moving nontargets away are two conditions that, as with placing the target in front of the nontarget, increase the target-nontarget relative saliency and lower the amplitude of the negativity produced by incongruity. Such negativities are therefore most likely to be specifically related to the processing of interference.

A nontarget can interfere with the processing of a target at different levels. It can first prime the incorrect response (Kopp et al., 1996) and consequently create response competition (Volberg \& Hübner, 2004). Such interference is normally accompanied by an increase in RTs and a frontocentral N2, specifically reflecting response competition (Han, Fan, Chen, \& Zhuo, 1999; Kopp et al., 1996; Van Veen \& Carter, 2002). Neither of these two effects was found in the present results, although the nontarget was associated with an incongruent response. Interference was strong when the target was occluded, and consequently, longer RTs, combined with frontocentral N2s, were there- fore expected. These effects were also foreseen between the nonoccluded target of the same-shape stimuli and the nonoccluded target of the different-shape stimuli, but to a smaller extent, given the small amount of influence that the nontarget had in these conditions. The results did not correspond to any of these expectations. This could be because of the further processing - probably amodal completion - of the target when it was occluded. This processing might have enhanced the perception of the target and contributed to the suppression of the nontarget, up to a point that prevented response completion. In other studies on interference in which hierarchical or flanker stimuli were used, targets were presented intact, and no such additional processing had to take place. Another explanation for the lack of RT differences and centrofrontal N2 effects on interference could pertain to the fact that the incongruous stimuli (i.e., same-shape stimuli) were compared with neutral stimuli (i.e., different-shape stimuli), rather than with congruent ones, as was the case in most studies in the literature. In such conditions of congruency, participants may pay more attention to the nontargets, which would shorten RTs. In conditions of incongruity, however, there would be response competition and, therefore, a lengthening of RTs.

Despite conditions favoring the perception of the target (i.e., amodal completion and the use of a neutral condition), a negativity to interference was found, which indicates monitoring of the nontarget. Because it was not accompanied by changes in RTs, this negativity (the N380) therefore most likely came after the primary visual analyses that are required to discriminate a square from a tilted square (Brodeur, Bacon, et al., 2008). These analyses are known to take place very early in the visual system (Bullier, 2001). At stimulus presentation, the visual system engages a series of operations that are responsible for extracting the stimulus contours and other features so as to build a schematic representation of the stimuli and to activate further processing (e.g., figure-ground segmentation) that will ultimately ensure the quality of the final visual output and contribute to the phenomenological aspects of perception. Only the early steps of this series may be necessary to discriminate shapes as simple as squares and tilted squares. The subsequent steps nevertheless take place in order to ensure visual perception of a quality corresponding to what we experience in everyday life. The latter processes demand more brain resources when there is interference. This interpretation has the advantage of accounting for the lack of reaction time delays and of frontocentral negativity in our data. It nevertheless requires further investigation to better define the brain processes at stake in the later stages.

\section{AUTHOR NOTE}

M.B.B. was supported by a fellowship from the Fonds de la Recherche en Santé du Québec (FRSQ), L.R. by Fellowship 13542 from the FRSQ, J.B.D. by Scholarship MSH-40304 of the Canadian Institute for Health Research, and F.L. by a Canadian Research Chair of the Natural Sciences and Engineering Research Council of Canada. The study was supported by Grant 96997-10 from the FRSQ allocated to J.B.D. Correspondence concerning this article should be addressed to M. B. Brodeur, Human Neurocognitive Science Lab, Douglas Mental Health University 
Institute, 6875 Boulevard LaSalle, Verdun, Québec, H4H 1R3 Canada (e-mail: mathieu.brodeur@gmail.com).

\section{REFERENCES}

American Electrophysiological Society (1994). Guideline thirteen: Guideline for standard electrode position nomenclature. Journal of Clinical Neurophysiology, 11, 111-113.

BeLL, A. J., \& SeJNowsKI, T. J. (1995). An information-maximization approach to blind separation and blind deconvolution. Neural Computation, 7, 1129-1159. doi:10.1162/neco.1995.7.6.1129

Boer, L. C., \& Keuss, P. J. (1982). Global precedence as a postperceptual effect: An analysis of speed-accuracy tradeoff functions. Perception \& Psychophysics, 31, 358-366.

Brodeur, M., Bacon, B. A., Renoult, L., Prévost, M., Lepage, M., \& Debruille, J. B. (2008). On the functional significance of the P1 and $\mathrm{N} 1$ effects to illusory figures in the notch mode of presentation. PLOS ONE, 3, e3505.

Brodeur, M., Lepore, F., Bacon, B. A., \& Debruille, J. B. (2009). Simultaneous completions of modal and amodal figures: Visual evoked potentials reveal asymmetrical interference effects. Visual Cognition, 17, 632-654. doi: $10.1080 / 13506280802003640$

Brodeur, M., Lepore, F., Lepage, M., Bacon, B. A., Jemel, B., \& Debruille, J. B. (2008). Alternative mode of presentation of Kanizsa figures sheds new light on the chronometry of the mechanisms underlying the perception of illusory figures. Neuropsychologia, 46, 554566. doi:10.1016/j.neuropsychologia.2007.10.001

Brown, J. M., \& Косн, C. (1993). Influences of closure, occlusion, and size on the perception of fragmented pictures. Perception \& Psychophysics, 53, 436-442.

Bullier, J. (2001). Integrated model of visual processing. Brain Research Review, 36, 96-107.

Delorme, A., \& MAKEIG, S. (2004). EEGLAB: An open source toolbox for analysis of single-trial EEG dynamics including independent component analysis. Journal of Neuroscience Methods, 134, 9-21.

Doniger, G. M., Foxe, J. J., Murray, M. M., Higgins, B. A., Snodgrass, J. G., Schroeder, C. E., \& JavitT, D. C. (2000). Activation timecourse of ventral visual stream object-recognition areas: High density electrical mapping of perceptual closure processes. Journal of Cognitive Neuroscience, 12, 615-621. doi:10.1162/089892900562372

Eriksen, B. A., \& EriKsen, C. W. (1974). Effects of noise letters upon the identification of a target letter in a non-search task. Perception \& Psychophysics, 16, 143-149.

GEgEnfurtner, K. R., Brown, J. E., \& RiEger, J. (1997). Interpolation processes in the perception of real and illusory contours. Perception, 26, 1445-1458. doi:10.1068/p261445

Gehring, W. J., Gratton, G., Coles, M. G., \& Donchin, E. (1992). Probability effects on stimulus evaluation and response processes. Journal of Experimental Psychology: Human Perception \& Performance, 18, 198-216. doi:10.1037/0096-1523.18.1.198

Greenhouse, S. W., \& Geisser, S. (1959). On methods in the analysis of profile data. Psychometrika, 24, 95-112.

Han, S., Fan, S., Chen, L., \& Zhuo, Y. (1999). Modulation of brain activities by hierarchical processing: A high-density ERP study. Brain Topography, 11, 171-183. doi:10.1023/A:1022244727182

HaN, S., \& HE, X. (2003). Modulation of neural activities by enhanced local selection in the processing of compound stimuli. Human Brain Mapping, 19, 273-281. doi:10.1002/hbm.10121
Han, S., He, X., Yund, E. W., \& Woods, D. L. (2001). Attentional selection in the processing of hierarchical patterns: An ERP study. Biological Psychology, 56, 113-130. doi:10.1016/S0301-0511(01)00073-4

Han, S., Song, Y., Ding, Y., Yund, E. W., \& Woods, D. L. (2001). Neural substrates for visual perceptual grouping in humans. Psychophysiology, 38, 926-935. doi:10.1111/1469-8986.3860926

Kellman, P. J., \& Shipley, T. F. (1991). A theory of visual interpolation in object perception. Cognitive Psychology, 23, 141-221. doi:10.1016/ 0010-0285(91)90009-D

Kopp, B., Rist, F., \& MattLer, U. (1996). N200 in the flanker task as a neurobehavioral tool for investigating executive control. Psychophysiology, 33, 282-294. doi:10.1111/j.1469-8986.1996.tb00425.x

Liotti, M., Woldorff, M. G., Perez, R., \& Mayberg, H. S. (2000). An ERP study of the temporal course of the Stroop color-word interference effect. Neuropsychologia, 38, 701-711. doi:10.1016/S0028 -3932(99)00106-2

Makeig, S., Jung, T. P., Bell, A. J., Ghahremani, D., \& Sejnowski, T. J. (1997). Blind separation of auditory event-related brain responses into independent components. Proceedings of the National Academy of Sciences, 94, 10979-10984.

Murray, M. M., Foxe, D. M., JavitT, D. C., \& Foxe, J. J. (2004). Setting boundaries: Brain dynamics of modal and amodal illusory shape completion in humans. Journal of Neuroscience, 24, 6898-6903. doi:10.1523/JNEUROSCI.1996-04.2004

NAVON, D. (1977). Forest before trees: The precedence of global features in visual perception. Cognitive Psychology, 9, 353-383. doi:10.1016/ 0010-0285(77)90012-3

Onton, J., Westerfield, M., Townsend, J., \& Makeig, S. (2006). Imaging human EEG dynamics using independent component analysis. Neuroscience \& Biobehavioral Reviews, 30, 808-822. doi:10.1016/ j.neubiorev.2006.06.007

PAQUeT, L. (1994). Does the attentional state determine processing dominance in to-be-ignored compound stimuli? Acta Psychologica, 85, 155-169. doi:10.1016/0001-6918(94)90030-2

Pegna, A. J., Khateb, A., Murray, M. M., Landis, T., \& Michel, C. M. (2002). Neural processing of illusory and real contours revealed by high-density ERP mapping. NeuroReport, 13, 965-968.

Stroop, J. R. (1935). Studies of interference in serial verbal reactions. Journal of Experimental Psychology, 18, 643-662.

VAn VeEn, V., \& CARTER, C. S. (2002). The timing of action-monitoring processes in the anterior cingulate cortex. Journal of Cognitive Neuroscience, 14, 593-602. doi:10.1162/08989290260045837

VolberG, G., \& HüBNER, R. (2004). On the role of response conflicts and stimulus position for hemispheric differences in global/local processing: An ERP study. Neuropsychologia, 42, 1805-1813. doi:10.1016/ j.neuropsychologia.2004.04.017

Wagemans, J., VAN Lier, R., \& Scholl, B. J. (2006). Introduction to Michotte's heritage in perception and cognition research. Acta Psychologica, 123, 1-19. doi:10.1016/j.actpsy.2006.06.003

Ward, L. M. (1983). On processing dominance: Comment on Pomerantz. Journal of Experimental Psychology: General, 112, 541-546. doi:10.1037/0096-3445.112.4.541

WEST, R., \& AlaIN, C. (1999). Event-related neural activity associated with the Stroop task. Cognitive Brain Research, 8, 157-164. doi:10.1016/ S0926-6410(99)00017-8

(Manuscript received August 18, 2009; revision accepted for publication March 24, 2010.) 